\section{5 \\ SCREENING BATTERIOLOGICO PER SGB IN DONNE GRAVIDE A TERMINE REALTÀ LOCALE}

\section{Ferrari L.}

Laboratorio di Microbiologia

Azienda Ospedale di Cremona

Introduzione. La presenza di Streptococcus agalactiae (SGB) sulla mucosa vaginale e/o rettale delle donne al termine della gravidanza, rappresenta un grave fattore di rischio infettivo per il nascituro.

Lo scopo della ricerca è stato verificare la presenza di SGB nei distretti vaginale e rettale delle pazienti gravide afferenti alla Divisione di Ostetricia dell'Ospedale di Cremona, al fine di determinarne la frequenza di colonizzazione. Alle donne risultate colonizzate da SGB in uno o in entrambe i distretti, (come da protocollo) è stato somministrato, durante il travaglio, un trattamento antibiotico profilattico a base di ampicillina per via parenterale. L'efficienza della profilassi è stata poi verificata valutando la frequenza con la quale gravide colonizzate da SGB hanno partorito neonati colonizzati a loro voltata.

Materiali e Metodi. E' stata condotta un'indagine retrospettiva sui dati microbiologici, (relativi alla presenza di SGB), provenienti da 5056 donne transitate per Divisione di Ostetricia dell'Azienda Istituti Ospitalieri. di Cremona nel periodo: gennaio 2001 - dicembre 2005 e sui neonati. I dati sono riferiti alle indagini colturali eseguite su: Tampone Vaginale (TV) e Rettale (TR) prelevati alle gestanti in 3536ma settimana di gravidanza e T.Auricolare (TA), T.Faringeo (TF) e T. Rettale (TR) prelevati ai nati partoriti da donne con almeno un tampone risultato positivo per SGB. I tamponi sono stati seminati su agar sangue Columbia+CNA (BD) e l'identificazione è stata eseguita mediante test in agglutinazione su vetrino (Oxoid)

Risultati e Conclusioni. L'analisi dei dati evidenzia 3697 gestanti $(73.1 \%)$ risultate negative per entrambe i tamponi, mentre $548(10.8 \%)$ presentano entrambe i campioni positivi. In 608 donne (12.0\%) si evidenzia positività del TR mentre risulta completamente negativo il TV. Solo 203 donne $(4.0 \%)$ presentano positività del TV non confermata dal TR, che risulta completamente negativo.

Globalmente in 1359 gestanti $(26.9 \%)$ è stato isolato SGB da almeno uno dei due tamponi effettuati, ciò si traduce in un elevato rischio di trasmissione di SGB al nascituro. La frequente positività del TR abbinata ad una negatività del TV (608 casi, pari al 12.0\%) sottolinea la necessità di effettuare sempre la ricerca di SGB su entrambe i distretti, onde evitare risultati falsamente negativi, non evidenziabili dall'esecuzione del solo TV.

Da 106 nati da madri positive (7.8\%), nonostante profilassi, è stato isolato SGB da almeno uno dei tre campioni biologici valutati (TA, TR, TF).

\section{6 \\ CANDIDOSI ORALE:ASPETTI CLINICI E MICROBIOLOGICI}

\author{
Gatti M., Rizzati T.G.,Almeria M.
}

Dipartimento di Scienze Odontostomatologiche, Sez. di Microbiologia, Alma Master Studiorum, Università degli Studi di Bologna Via S.Vitale 59, 40I 25 Bologna.

Introduzione. I miceti del genere Candida sono commensali del cavo orale. Nell'ultimo decennio le infezioni del cavo orale sono aumentate come incidenza, sia per l'aumentata richiesta di diagnosi di infezioni orali da parte dei clinici sia per la pandemia sostenuta dall'AIDS. Scopo del presente lavoro è stato rivolto alla ricerca di Candida spp. in soggetti presunti sani per rilevarne l'incidenza, individuare le specie più frequenti per attuare un corretto protocollo di igiene orale intento a prevenire le manifestazioni cliniche.

Metodi. Sono stati analizzati 47 prelievi microbiologici ottenuti attraverso l'utilizzo di tamponi della mucosa orale o del palato di 47 partecipanti, suddivisi in due gruppi: gruppo I comprendente 14 pazienti con età maggiore di 65 anni; gruppo II comprendente 33 pazienti con età inferiore ai 65 anni. Previa osservazione microscopica diretta i campioni sono stati seminati su piastre di Sabouraud Dextrose agar addizionato di cloramfenicolo (Biolife) e/o Chromoagar (Alfa-Wasserman) e incubati a $37^{\circ} \mathrm{C}$ in aerobiosi per un massimo di quattro giorni. Le colonie cresciute su entrambi i terreni venivano identificate mediante test di filamentazione e prove biochimiche di assimilazione di carboidrati API 20C AUX (bioMerieux). Per Candida dubliniensis è stata verificata anche la negatività della crescita delle colonie sia a $42^{\circ} \mathrm{C}$ sia a $45^{\circ} \mathrm{C}$ ed in brodo con il $6.5 \%$ di NaCl.

Risultati. Hanno evidenziato che il 38\% del campione totale era positivo nei confronti dei miceti del genere Candida e il $62 \%$ era negativo. Nel gruppo I i pazienti positivi erano il $43 \%$ e sono state identificate le specie:

C. albicans e C. glabrata. Nel gruppo II i pazienti positivi erano il $33 \%$ e sono state identificate le specie: C. albicans, C. dubliniensis e C. tropicalis.

Conclusioni. Nonostante le numerose specie di Candida che sono state isolate, la specie predominante è risultata essere C. albicans, identificata anche in campioni che proponevano fattori di rischio confermati dalla letteratura, quali presenza di una protesi totale removibile e diabete mellito. I pazienti positivi sono stati sottoposti ad un preciso protocollo di igiene orale che prevedeva: incontri motivazionali atti all'informazione circa i rischi e le possibili complicazioni dovute alla presenza del microrganismo identificato; istruzioni circa l'igiene orale domiciliare e l'igiene e la cura di eventuali protesi removibili; detartrasi; rivalutazione della compliance a 3 e 6 mesi. 